\title{
Time course of ECG depolarization and repolarization changes during ischemia in PTCA recordings
}

\author{
E. Pueyo ${ }^{1}$, J. García $^{1}$, G. Wagner ${ }^{2}$, R. Bailón ${ }^{1}$, L. Sörnmo ${ }^{3}$, P. Laguna ${ }^{1}$ \\ ${ }^{1}$ Department of Electronic Engineering and Communications, University of Zaragoza, Zaragoza, Spain. \\ ${ }^{2}$ Department of Medicine, Duke University Medical Centre, NC, USA. \\ ${ }^{3}$ Department of Applied Electronics, University of Lund, Sweden
}

\begin{abstract}
In this work we study the temporal evolution of changes in the electrocardiogram (ECG) as a consequence of the induced ischemia during prolonged coronary angioplasty. We compare the time course of indexes reflecting depolarization and repolarization respectively, considering both local (measured in specific points of the ECG) and global (obtained from the Karhunen-Loève transform) indexes. In particular, the evolution of Q, R and $\mathrm{S}$ wave amplitudes during ischemia is analysed with respect to classical indexes such as ST level. As a measurement of sensitivity we use an Ischemic Changes Sensor (ICS), which reflects the capacity of an index to detect changes in the ECG. The results show that, in leads with low-amplitude ST-T complexes, the $\mathrm{S}$ wave amplitude is more sensitive in detecting ischemia than is the commonly used index ST60. It is found that the S wave amplitude in such leads initially exhibits a delayed response to ischemia, when compared to ST60, but is better in detecting ischemia from the second minute of occlusion. The global indexes describing the ST-T complex are, in terms of the ICS, superior to the $\mathrm{S}$ wave amplitude in detecting ischemia.

Keywords: Ischemia, Angioplasty, QRS amplitudes, ST value.
\end{abstract}

\section{INTRODUCTION}

Ischemic changes in the electrocardiogram (ECG) may precede anginal pain. Thus it is important to search for ECG-based indexes with good capability for the detection of ischemia. Percutaneous Transluminal Coronary Angioplasty (PTCA), consisting in a sudden complete coronary occlusion produced by balloon angioplasty, is a useful model to study ischemic changes that occur during the first minutes of occlusion.

Several studies have reported both ST segment and QRS complex changes evoked by PTCA even when the occlusion is not prolonged [1-5]. In other studies, indexes based on the Karhunen-Loève transform have been developed which reflect information contained in an entire ECG segment or waveform. It has been shown that these global indexes show a higher sensitivity and an earlier response in the detection of ischemia than the local indexes [6-7]. Other proposals have suggested the study of the ECG during ischemia by means of a new index (the Athens score) that assembles the variations of $\mathrm{Q}, \mathrm{R}$ and $\mathrm{S}$ wave amplitudes [8].

In this study we analyse several clinical indexes from depolarization (in particular, Q, R and $\mathrm{S}$ wave amplitudes) and compare them with other indexes from repolarization by measuring their capacity to reflect the ischemic changes induced during prolonged PTCA according to the methodology proposed in [7].

\section{MATERIALS AND METHODS}

\subsection{Study population}

The study group consisted of 83 patients ( 55 males, 28 females) from the STAFFIII database receiving elective PTCA in one of their major coronary arteries. The average duration of the occlusion period was 4' 26", considerably longer than that of usual PTCA procedures because the treatment protocol included a single prolonged occlusion rather than a series of brief occlusions.

The locations of the 83 dilations were: left anterior descending artery (LAD) in 27 patients, right coronary artery (RCA) in 38 patients and left circumflex artery (LCX) in 18 patients. Nine standard leads (V1-V6, I, II and III) were recorded using equipment by SiemensElema AB (Solna, Sweden) and digitized at a sampling rate of $1 \mathrm{KHz}$ and amplitude resolution of $0.6 \mu \mathrm{V}$.

For each patient in the study group two recordings were considered: the control ECG recorded just before angioplasty and the PTCA recording. The control interval usually was 5 minutes long, the same order as the average inflation time.

\subsection{ECG-based indexes}

ECG signal pre-processing was applied before measuring the different parameters, including the detection and selection of 'normal beats' labelled 
according to [9], cubic splines baseline wander attenuation, rejection of beats presenting differences in their mean isoelectric level respect to adjacent beats of more than $600 \mu \mathrm{V}$ and signal averaging using a moving window of 10 beats (only prior to measure the local indexes) to improve the signal-to-noise ratio.

The onset and offset of the different ECG waveforms were detected by using the automated detector of waveform boundaries described in [10].

On the pre-processed signal we considered several indexes measured on specific ECG points (or derived from two ECG points in the case of interval durations): the level of the ST segment measured at $60 \mathrm{~ms}$ after $\mathrm{J}$ point (ST60), the maximum amplitude of the $\mathrm{T}$ wave (Ta) and its corresponding time location with respect to $\mathrm{R}$ wave (Tp), the QT interval length (QT) and the amplitudes of $\mathrm{Q}, \mathrm{R}$ and $\mathrm{S}$ waves $\left(\mathrm{Q}_{\mathrm{a}}, \mathrm{R}_{\mathrm{a}}\right.$ and $\left.\mathrm{S}_{\mathrm{a}}\right)$.

These indexes were compared with other global indexes derived from the Karhunen-Loève transform (KLT). The KLT captures the information contained in an entire signal segment and concentrates it in a few coefficients. This transform was applied to the QRS complex and the entire ST-T complex. For each of these intervals the first four KLT series were considered and then the one that exhibited the largest changes (in the sense of greatest variations at the end of the occlusion averaged among the 83 patients and the 12 leads) was selected. These parameters are denoted by $\alpha^{\text {QRS }}$ and $\alpha^{\text {STT }}$. A detailed development of the KLT and how this is applied to the ECG segments can be found in [6].

\subsection{The Ischemic Changes Sensor}

With the two recordings considered for each patient, before and during the angioplasty process, an Ischemic Changes Sensor (ICS) is defined. This parameter, described in [6-7], reflects the capacity of a certain index to detect the changes induced by ischemia and is obtained according to the following formula:

$$
I C S_{\text {index }}(t)=\frac{\Delta \text { index }(t)}{\sigma_{\text {index }}}
$$

Aindex(t) is the magnitude of a change reflected in the index during the occlusion, estimated at an instant $t$ of the occlusion by fitting a linear polynomial to the series values of the index observed from the beginning of the inflation until the instant $t$ considered. Calculating $\Delta i n d e x$ in this way, we avoid outlier measures. $\sigma_{\text {index }}$ is the standard deviation of the index in the control recording and measures the normal variations of the index when no occlusion is present. Finally, index represents any of the indexes considered in the study.
To investigate the time course of the ischemic changes, the ICS was estimated every 10 seconds from the beginning of the occlusion.

To study the significance of an index for ischemia detection, a threshold $\eta$ was applied to the ICS parameter. Such a threshold was selected by considering the ICS values measured in a control population composed of 11 normal subjects without any symptoms of cardiac diseases. The ICS values for those subjects were not larger than 2.5 in any case. In order to ensure that only ischemic events were detected, a considerably larger threshold value was used $(\eta=8)$.

\section{RESULTS AND DISCUSSION}

First, we studied the changes shown by the different indexes evaluated at the end of the occlusion (see Fig. 1).

When the $\mathrm{Q}_{\mathrm{a}}, \mathrm{R}_{\mathrm{a}}$ and $\mathrm{S}_{\mathrm{a}}$ indexes from ventricular depolarization were not included in the study, the ST60 index was the most sensitive of the traditional indexes in all the leads [6]. However, when these three indexes were also considered in this study, we found that in leads with usual low-amplitude ST-T complexes, such as V6, aVF, II or III, the mean of the final ICS (in absolute value) among the 83 patients was larger for the index $S_{a}$ than for ST60 (see Fig. 1).

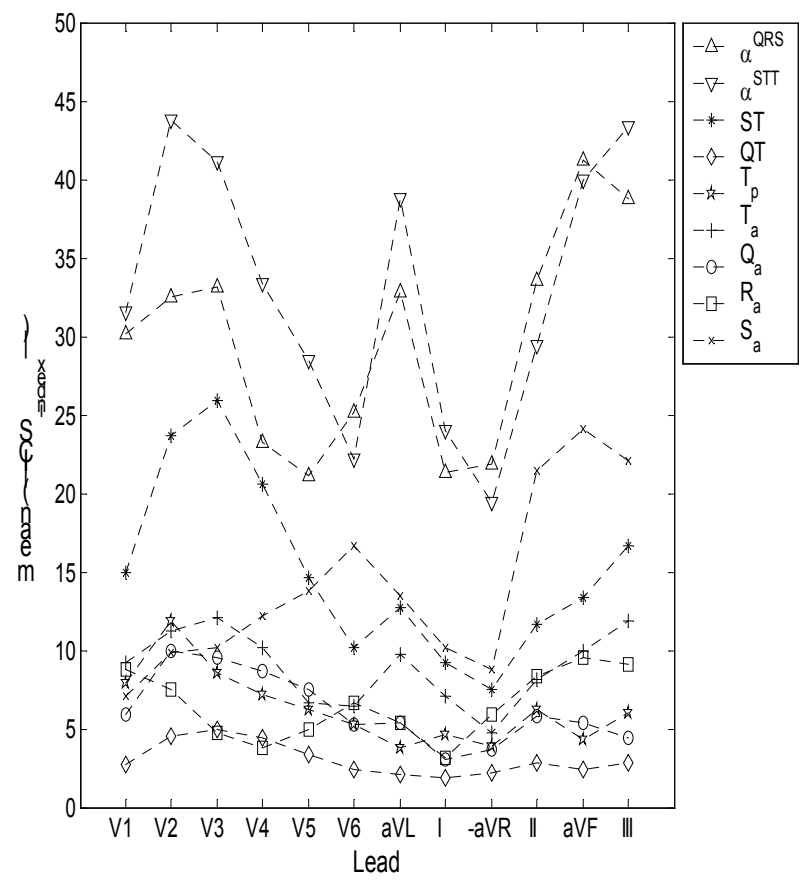

Fig. 1. Mean of the absolute value of ICS [ mean ( $\mid$ ICS | ) ] among the 83 patients for the different indexes at the end of the occlusion in each lead. 
The leads V2, V3 and V4 were the most sensitive ones (in the sense of largest values of mean [ | ICS | ] ) for detection of induced ischemia when this matter was analysed by means of the ST60 index. When this consideration was established in terms of $\mathrm{S}$ wave amplitude, the leads aVF, II and III proved to be the most sensitive ones.

Only the ICS values corresponding to the two abovementioned parameters, $\mathrm{ST}$ and $\mathrm{S}_{\mathrm{a}}$, approach to the range of values of the indexes derived from the KL transform. The other two indexes added in this study, $\mathrm{Q}_{\mathrm{a}}$ and $\mathrm{R}_{\mathrm{a}}$, exhibit values of change considerably lower, similar to those associated with other local indexes already considered, such as Tp.

Next, the temporal evolution of changes, registered by measuring the ICS at different times of the occlusion, is analysed. Only the performance of $\mathrm{S}_{\mathrm{a}}$ among the three new indexes included in the study is remarkable, since $\mathrm{ICS}_{\mathrm{Qa}}$ and $\mathrm{ICS}_{\mathrm{Ra}}$ did not show significant changes during the occlusion, taking similar values to the ones found for $\mathrm{ICS}_{\mathrm{Tp}}$ (see Fig. 2 and Fig. 3).

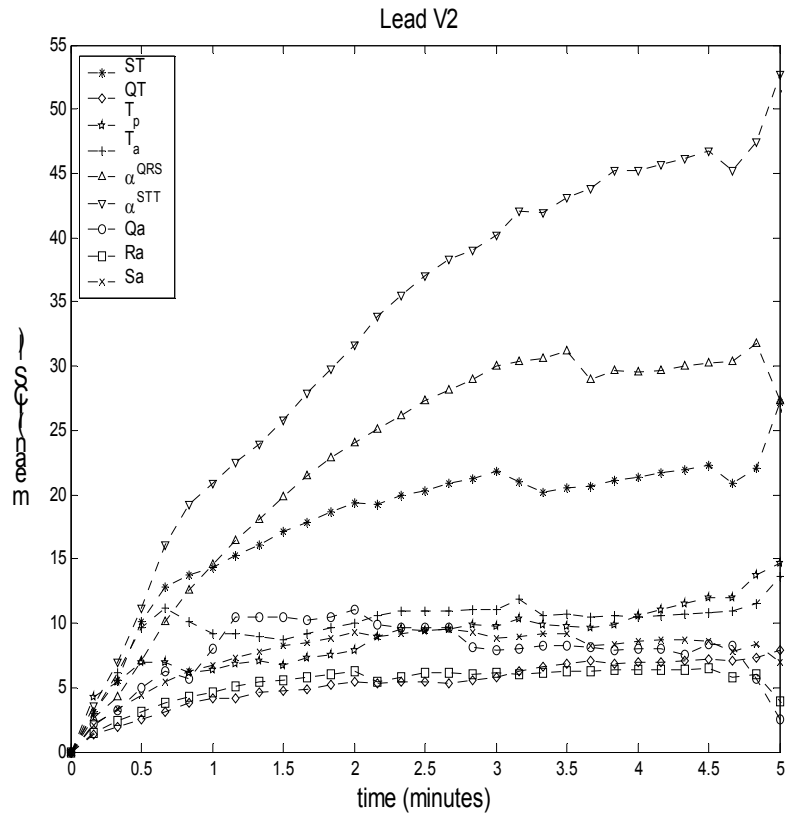

Fig. 2. Temporal evolution of the different ICS averaged among patients for the lead V2. Due to the differences in the occlusion time the number of patients included in the average changes for different times.

The $\mathrm{S}_{\mathrm{a}}$ index, which shows much more noticeable variations, presents different performances depending on whether the projection of the ST-T complex on the lead in consideration is large or small with respect to what occurs in the remaining leads. Thus, in leads V2, V3 and $\mathrm{V} 4$ the range of ICS values taken by $\mathrm{S}_{\mathrm{a}}$ is similar to the one corresponding to $\mathrm{Ta}$, being these values larger than those found for the rest of local indexes such as Tp and QT, but, in any case, much smaller than the ones referred to ST (see Fig. 2).

However, in other leads such as aVF, II, V5, V6, the changes registered by $S_{a}$ are much more noticeable. In fact, although $\mathrm{ICS}_{\mathrm{Sa}}$ has a slow response in comparison with $\mathrm{ICS}_{\mathrm{ST}}$, we can find an instant (2 minutes in V5, 1.5 minutes in $\mathrm{aVF}, \ldots)$ in which $\mathrm{ICS}_{\mathrm{Sa}}$ reaches the value taken by $\mathrm{ICS}_{\mathrm{ST}}$ and from then on remains higher until the end of the occlusion (see Fig. 3). Nevertheless, the local indexes always exhibit a change that is less pronounced than that of the indexes based on the KL transform [7].

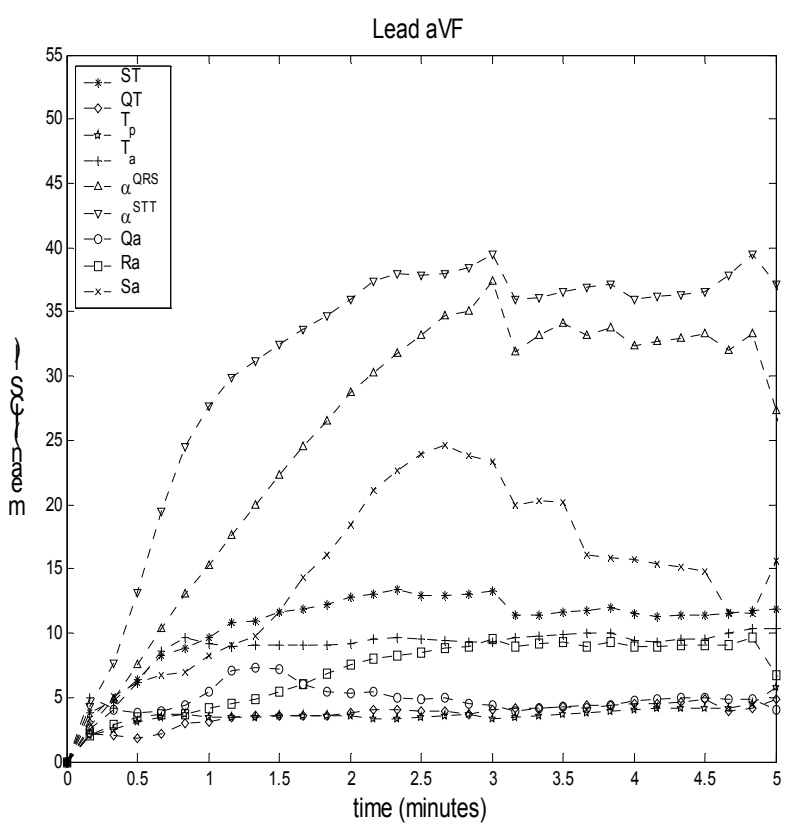

Fig. 3. Temporal evolution of the different ICS averaged among patients for the lead aVF.

The results obtained when $\mathrm{S}_{\mathrm{a}}$ parameter is introduced corroborate the idea that the indexes related to the depolarization (QRS complex) react to the induced ischemia later in time than the indexes referred to the repolarization (ST-T complex). In fact, if we evaluate the contribution to the final ICS value for each of the indexes considered in the study, we can observe that for indexes associated with the ST-T complex (such as the ST60 index), the ICS grows very fast during the first minute of occlusion, but this increase is much slower during the remaining minutes. In contrast, for depolarization indexes such as $\mathrm{S}_{\mathrm{a}}$ it is necessary to wait until the second minute to reach a large percentage of the final ICS.

Finally, we study the percentage of patients who had their earliest ischemic changes in one of the following three intervals of the ECG: ST segment, T wave and QRS complex. 


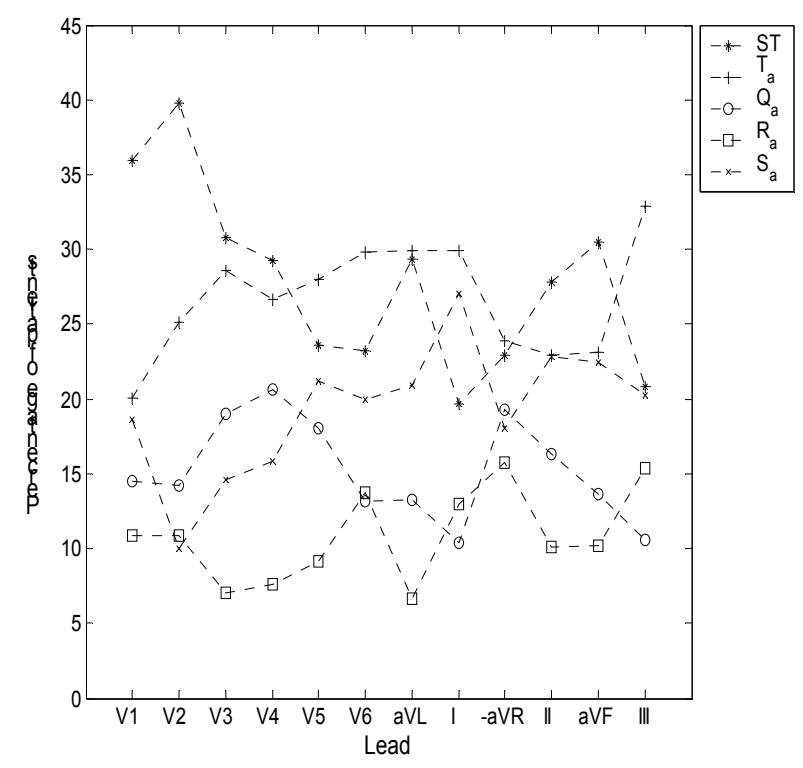

Fig. 4. Percentage of patients in whom the different indexes showed the earlier response to the ischemic induced changes, considering the indexes $\mathrm{Q}_{\mathrm{a}}, \mathrm{R}_{\mathrm{a}}, \mathrm{S}_{\mathrm{a}}$ from depolarization and $\mathrm{ST}$, $\mathrm{T}_{\mathrm{a}}$ (the most significant ones) from repolarization.

The results we have obtained support the idea of an earlier response of the indexes related to the repolarization with respect to the ones from depolarization, although it is remarkable that when the new variables are included (in particular $S_{a}$ ), the percentage of patients with their earliest changes in QRS complex has a considerable increase (see Fig.4).

\section{CONCLUSIONS}

The ECG changes induced by ischemia occur both at repolarization and depolarization. In this study we have evaluated depolarization changes compared to repolarization ones. We found that in some leads (those with usual lower projection of the ST segment) the S wave amplitude $\left(S_{a}\right)$ reaches rates of change superior to the ones corresponding to the ST60. The variations of this index $\mathrm{S}_{\mathrm{a}}$ based on the depolarization occur later in time than those corresponding to indexes associated with the repolarization, which have an earlier response.

The changes given by local indexes, both at depolarization and repolarization, do not reflect by themselves all the changes induced by ischemia in the ECG. It has been shown that there are other indexes obtained from the KL transform that are more sensitive in the detection of ischemia and exhibit an earlier activation to it than the local ones.

The results we have obtained support the idea of the 'ischemic cascade', according to which the ischemic perturbations in the QRS are preceded by changes in the $\mathrm{T}$ wave and in the ST segment.

\section{ACKNOWLEDGEMENTS}

This work was supported by projects P075/2001 CONSID-DGA and TIC2001-2167-C02:02, Spain.

\section{REFERENCES}

[1] F. Kornreich, R.S. Macleod, V. Dzavik et al, "QRST changes during and after percutaneous transluminal coronary angioplasty," J. Electrocardiol, pp. 27:113, 1994.

[2] N.G. Wagner, D.C. Sevilla, M.W. Krucoff et al, "Transient alterations of the QRS complex and ST segment during percutaneous transluminal balloon angioplasty of the left anterior descending coronary artery," Am. J. Cardiol., pp. 62:1038, 1988

[3] S. Abboud, J.M. Smith, B. Shargorodsky et al, "High frequency electrocardiography of three orthogonal leads in dogs during a coronary artery occlusion," PACE, pp. 12:521, 1989.

[4] S. Abboud, R..J. Cohen, A. Selwyn et al, "Detection of transient myocardial ischemia by computer analysis of standard and signal-averaged high-frequency electrocardiograms in patients undergoing percutaneous transluminal coronary angioplasty," Circulation, pp. 76:585, 1987.

[5] J. Petterson, S. Warren, N. Mehra et al, "Changes in highfrequency QRS components during prolonged coronary artery occlusion in humans," J Electrocardiol., pp. 28:225, 1995.

[6] J. García, P. Lander, L. Sörnmo, S. Olmos, G. Wagner, and P. Laguna, "Comparative study of local and KarhunenLoève based ST-T indexes in recordings from human subjects with induced myocardial ischemia," Comput. Biomed. Res., vol. 31, no 4, pp 271-292, 1998.

[7] J. García, G. Wagner, L. Sörnmo, S. Olmos, P. Lander, and P. Laguna, "Temporal evolution of traditional vs. transformed ECG-based indexes in patients with induced myocardial ischemia," Journal of Electrocardiol., vol. 33, no 1, pp 37-47, 2000.

[8] A.Michaelides, F. Triposkiadis, H. Boudoulas, et al, "New coronary artery disease index based on exercise-induced QRS changes," Am. Heart J., 120(2), pp. 292-302, 1990.

[9] G.B. Moody, R.G. Mark, "Development and evaluation of a 2-lead ECG analysis program," Computers in Cardiology. IEEE Computer Society Press, pp. 39-44, 1982.

[10] P. Laguna, R. Jané, P. Caminal. "Automatic detection of wave boundaries in multilead ECG signals: Validation with the CSE database," Compu.t Biomed. Res., vol. 27(1) pp. 45-60, 1994.

Esther Pueyo Paules

C.P.S. María de Luna 3, 50015 Zaragoza, España epueyo@posta.unizar.es 